\title{
Purine and Pyrimidine-Linked Enzymes and Genes are Strongly Responsible for the Development of Tumors, Particularly Glioblastoma Multiforme
}

\author{
Kristine Edgar Danielyan 1*, Tatul Ashot Yeghiazaryan1, Samvel Grigoriy Chailyan1, \\ Levon Ruben Harutyunyan'2, Ruben Levon Harutyunyan², Gurgen Sergei Petrosyan ${ }^{3}$ \\ ${ }^{1} \mathrm{H}$. Buniatian Institute of Biochemistry, National Academy of Science of Armenia, Yerevan, Armenia \\ ${ }^{2}$ National Center of Oncology named after V. A. Fanarjian, Yerevan, Armenia \\ ${ }^{3}$ Institute for Informatics and Automation Problems of the National Academy of Science of Armenia, Yerevan, Armenia \\ Email: *Kristine_danielyan@biochem.sci.am
}

How to cite this paper: Danielyan, K.E., Yeghiazaryan, T.A., Chailyan, S.G., Harutyunyan, L.R., Harutyunyan, R.L. and Petrosyan, G.S. (2020) Purine and Pyrimidine-Linked Enzymes and Genes are Strongly Responsible for the Development of Tumors, Particularly Glioblastoma Multiforme. Computational Molecular Bioscience, 10, 73-80.

https://doi.org/10.4236/cmb.2020.103005

Received: July 6, 2020

Accepted: September 5, 2020

Published: September 8, 2020

Copyright $\odot 2020$ by author(s) and Scientific Research Publishing Inc. This work is licensed under the Creative Commons Attribution International License (CC BY 4.0).

http://creativecommons.org/licenses/by/4.0/

\begin{abstract}
We were aiming to delineate, by the utility of the biological data results, in our investigations the link between the purine and pyrimidine metabolism and development of the glioblastoma. We analyzed the sets of the genes, belonging to the purine and pyrimidine metabolism by the utility of GSEA software as well as MSIgnDB application of the GSEA. The GEO database, GEOR2 tools were serving for the visualization of the genes expression profiles of the disease. The Cancer Proteome Atlas as well as the tools of the data sets were also used to collect and analyze the results. We concluded and came to the following consequential results. 1) Neurogenesis and Glioblastoma are sharing some common genes. 2) Purine and pyrimidine metabolism-linked enzymes and genes are responsible for the upregulation of DNA and mRNA synthesis in the settings of the tumor development. 3) EGFR expression responsible genes, mRNA as well as protein is upregulated during the development of the glioblastoma. 4) GMPS genes are more strongly upregulated in the settings of the glioblastoma than ADSL. 5) PRPS1 is strongly synthetized in neurospheres in contrast to the mature tissue during glioblastoma development.
\end{abstract}

\section{Keywords}

Data Bases, Purine, Pyrimidine, Metabolism, Glioblastoma, PRPS1 


\section{Introduction}

We were aiming to delineate in our investigations, by the utility of the biological data results, the link between the purine and pyrimidine metabolism and development of glioblastoma.

In our previous experiments, we have proved experimentally, by the inhibition of the Xanthine Oxidoreductase (EC 1.17.3.2), the one of the last and the key regulative enzymes in the chain of the purines catabolism-responsible enzymes, by its classical compound-Allopurinol, it is possible to decrease the speed of purines decay [1] [2]. In addition, we have proved, by impacting the XOR, it is possible to accelerate the cells proliferation or have an influence on the cells' deaths [2] [3] [4].

The group of the scientists in 2017 published the data, proving the elevation of purine metabolism in the brain tumor initiating cells (BTICs) is high, in contrast to the already differentiated glioma cells (DGCs). Using immunoblot analysis, the authors also concluded, the expression levels of three purine synthesis-responsible enzymes, PRPS1 (Phosphoribosyl pyrophosphate synthetase 1; EC 2.7.6.1), ADSL (Adenylosuccinatelyase; EC 4.3.2.2.) and GMPS (GMP synthetase EC 6.3.5.2) - each representing an arm of the purine biosynthesis pathway-were significantly elevated in BTICs (Brain Tumor Initiating Cells) as compared to matched DGCs (Differentiated Glioma Cells) [5].

During our recent experiments we injected primer human glioblastoma cells (island-like spheres groups of the glioblastoma cells culture) into the rats' temporal cortex. At day $21^{\text {st }}$ AdenosineDeaminase2 (ADA2; EC 3.5.4.4), PRPS1 as well as the XOR activities were measured. Interestingly, activities of the mentioned enzymes were elevated [6].

The purpose of our current study was the globalized analyses of the involvement of the purine and pyrimidine metabolism responsible compounds and enzymes in the processes of the glioblastoma development.

\section{Methods}

We analyzed the sets of the genes, belonging to the purine and pyrimidine metabolism by the utility of GSEA software (on-line version, https://www.gsea-msigdb.org/gsea/index.jsp), Molecular Signature Databases (MSIgnDB) application of the GSEA (https://www.gsea-msigdb.org/gsea/msigdb). The GEO database, GEOR2 tools were serving for the visualization of the genes expression profiles of the disease. The Cancer Proteome Atlas as well as the tools of the data sets also were used to collect and analyze the results, including glioblastoma elevated and specific gene profile, mRNA and proteins (https://tcpaportal.org/tcpa/).

The graphs and tables, built by the programs, were replaced with the manually drawn to increase the resolution.

\section{Results}

1) First we extracted the genes from data sets, responsible for the purines' and 
pyrimidines' metabolism. After all, we isolated from those sets of the genes, that particular genome units, which were involved into the cancer genes data sets.

The existence of the numerous cancer-linked genes of the purine and pyrimidine catabolism was providing the fact of strong correlation between metabolic mentioned processes and cancer development. In addition, we drew out the KEGG pathways and sets of the genes of KEGG pathways, which were involving that particular genes as the cancer modules (Table 1). It is necessary to mention, we withdrew from GSEA 98 genes for pyrimidine metabolism, 159-from purine. After summarizing the purine and pyrimidine genes and collection them in the MSIgnDB window we applied cancer related "filters". Analyzing the results after application of the filters, we concluded, 91 genes from 257 were involved in the processes of cancer development.

We concluded, the predominant parts of those pathways are related to the RNA, DNA syntheses as well as the DNA repair in the settings of the cancer development.

2) Then we isolated the common genes (RRM1, POLR2I, POLE3, POLA2) from the purine metabolism vs gene neighborhoods network and pyrimidine metabolism vs gene neighborhoods and found the KEGG pathways, where these genes were involved, in the settings of the cancer development (Table 2).

Table 1. Utility of the KEGG pathways data sets to delineate the additional genes' role of purine and pyrimidine metabolism in the setting of cancer development.

\begin{tabular}{|c|c|c|c|c|}
\hline Description & $\begin{array}{l}\text { \# Genes in } \\
\text { Overlap (k) }\end{array}$ & $\mathrm{k} / \mathrm{K}$ & p-value & FDR q-value \\
\hline Purine metabolism & 43 & & $4.12 \mathrm{e}^{-97}$ & $7.66 \mathrm{e}^{-95}$ \\
\hline Pyrimidine metabolism & 30 & & $1.25 \mathrm{e}^{-66}$ & $1.16 \mathrm{e}^{-64}$ \\
\hline DNA replication & 8 & & $1.6 \mathrm{e}^{-16}$ & $9.91 \mathrm{e}^{-15}$ \\
\hline RNA polymerase & 6 & & $1.88 \mathrm{e}^{-12}$ & $8.73 \mathrm{e}^{-11}$ \\
\hline Drug metabolism-other enzymes & 6 & & $6.96 \mathrm{e}^{-11}$ & $2.59 \mathrm{e}^{-9}$ \\
\hline Base excision repair & 5 & & $1.06 \mathrm{e}^{-9}$ & $3.3 \mathrm{e}^{-8}$ \\
\hline Nucleotide excision repair & 5 & & $3.53 \mathrm{e}^{-9}$ & $9.37 \mathrm{e}^{-8}$ \\
\hline Huntington's disease & 6 & & $1.47 \mathrm{e}^{-7}$ & $3.43 \mathrm{e}^{-6}$ \\
\hline Vascular smooth muscle contraction & 5 & & $4.66 \mathrm{e}^{-7}$ & $9.59 \mathrm{e}^{-6}$ \\
\hline One carbon pool by folate & 2 & & $2.32 \mathrm{e}^{-4}$ & $4.04 \mathrm{e}^{-3}$ \\
\hline
\end{tabular}

Table 2. Utility of the KEGG pathways data sets to delineate the additional common genes' role of purine and pyrimidine metabolism in the setting of cancer development.

\begin{tabular}{ccccc}
\hline Description & \# Genes in Overlap (k) & $\mathrm{k} / \mathrm{K}$ & $\mathrm{p}$-value & FDR q-value \\
\hline Pyrimidine metabolism & 4 & & $3.99 \mathrm{e}^{-11}$ & $7.41 \mathrm{e}^{-9}$ \\
Purine metabolism & & $2.83 \mathrm{e}^{-10}$ & $2.63 \mathrm{e}^{-8}$ \\
DNA replication & & & $5.12 \mathrm{e}^{-6}$ & $3.17 \mathrm{e}^{-4}$ \\
\hline
\end{tabular}


It's logical and the results are evidencing that in the cancer-linked environment these genes are predominantly responsible for DNA replication.

3) Then we found the specific genes up which were regulated only during the Glioblastoma from the Human protein atlas.

These genes were transferred into the GSEA software for the further analyses. We found, these genes were involved into the data sets of the other sets of the genes, responsible mostly for several biological processes such as formation of the synapses and neurogenesis, which are evidencing about the common mechanism between the regenerative neurogenesis and glioblastoma development. Some sets of glioblastoma development genes are responsible for the establishment of the cell-cell signaling and formation of the synapsis, regulation of the nervous system development, ions transmembrane transporting and, finally, neurogenesis.

Interestingly, in our previous publications, we proved, the activation of the PRPS1 in the settings of experimental stroke modeling might increase the number of the Ki-67 positive cells in the region of the hippocampus [7].

4) Further, we analyzed the mRNA existence profile from the Cancer Proteome Atlas in the settings of glioblastoma. We found 6 mRNA. MRNA and genes from that particular subpart of the data sets which are Annexin 1, Caveolin 1, CKIT, EGFR, EGFR_pY1068, P16INK4A. These are mostly the structural and EGF related proteins.

It is very well know, EgfR (Egf Receptor) activation initiates formation of the tumors, different types of it, moreover, has a crucial role in progression of the metastasis [8]. EgfR are responsible for the triggering of the following pathways: Ras/Raf signaling cascade, which promotes cell survival and cell proliferation; phosphatidylinositol 3-kinase/Akt signaling cascade, responsible for cell growth, apoptosis resistance, cell invasion and migration; signal transducers and activators of transcription (STAT) pathway, inducing tumor progression, oncogenesis and angiogenesis; phospholipase $\mathrm{C} \gamma$ signaling regulation of ion channels, which induces cell migration, calcium-mediated signaling; Nck/PAK signaling cascade cell, responsible for the survival and cell migration; Cbl mediated endocytosis, inducing respectfully endocytotic processes [9]-[14].

Interestingly, EGF and EGFR activates the MAP kinase pathways, which in its own turn, by the process of phosphorylation activates the CAD (carbamoylphosphatesynthetase-aspartate carbamoyltransferase-dihydroorotase). It is the multifunctional protein, triggering the mammalian pyrimidine biosynthesis [15] [16]. Zollner et al. proved, by influencing on the pyrimidine metabolism, it is possible to have an impact on the purine metabolic pathways [17].

5) We worked also with the GEO data set and analyzed the profile of expression of the PRPS1 enzymes responsible for the syntheses of purines and pyrimidines.

We have chosen analysis of primary neurosphere cultures of glioblastoma (GBM) or oligodendroglial (ODG) tumors from 6 patients study (GDS4473). Patient-derived glioma-propagating cells (GPC) contain karyotypic and gene expression profiles that are found in the primary tumor. 
From the bottom of the page of the particular study (GDS4473) we chose GEOR tools. By "define" window the comparison groups were divided into the two folders of genes "oligodendroglialglioma" vs "neurospheres" in Homo sapiens. The "Value Distribution" window allowed to compare the quantity related homogeneity of the groups to be compared. "Top 250" allowed the comparison of the genes. Any genes distribution in the groups might be compared from the list of the entire sets of the genes.

Two sets of the results were analyzed regarding PRPS1 gene expression in glioblastoma multiforme and oligodendroglialglioma vsneurospheres in Homo sapiens. The GEO data results are evidencing about the up regulation of PRPS1 in neurospheres fraction in comparison with the already formed and maturated glioblastoma tissue (Figure 1).

By the utility of GEO2R we came to the conclusion, another two key regulative enzymes of purine and pyrimidine metabolism were up regulated in the glioblastoma multiforme tissues in comparison with the normal brain fractions.

Interestingly, from those two enzymes GMPS was more prominently unregulated in comparison with the ADSL (Figure 2).

\section{Discussion}

Glioblastoma (GBM) is the most common primary malignant brain tumor, comprising $16 \%$ of all primary brain and central nervous system neoplasms [18]. The average age-adjusted incidence rate is 3.2 per 100,000 population Originally, GBMs were thought to be derived solely from glial cells; however, evidence suggests
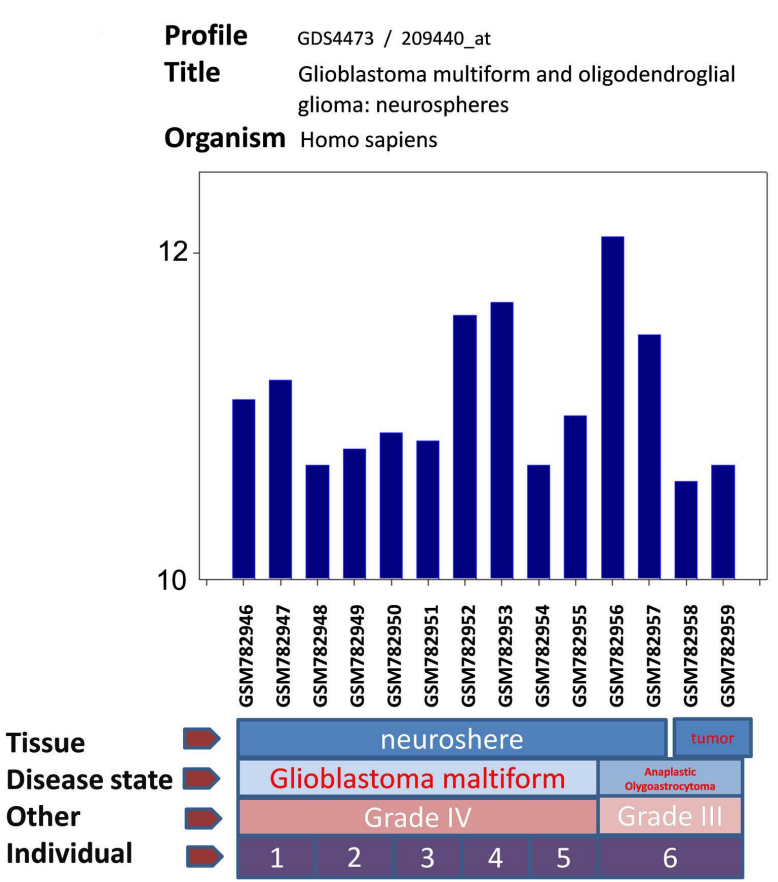

(a)
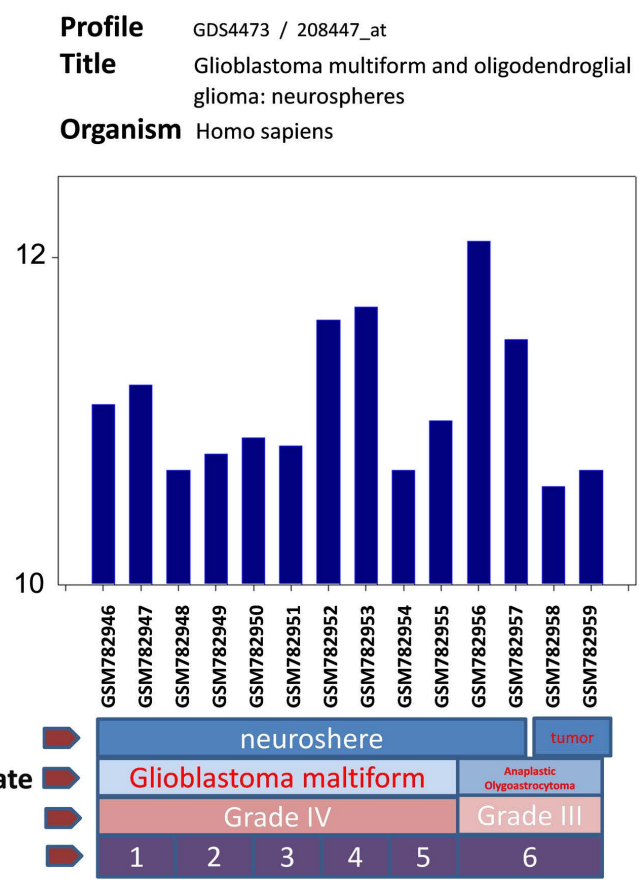

(b)

Figure 1. Comparison of the expression of the PRPS1 gene in glioblastoma multiforme and oligodendroglial gliomavs neurospheres in Homo sapiens in two sets of the results. Results are downloaded from GEO data base. 


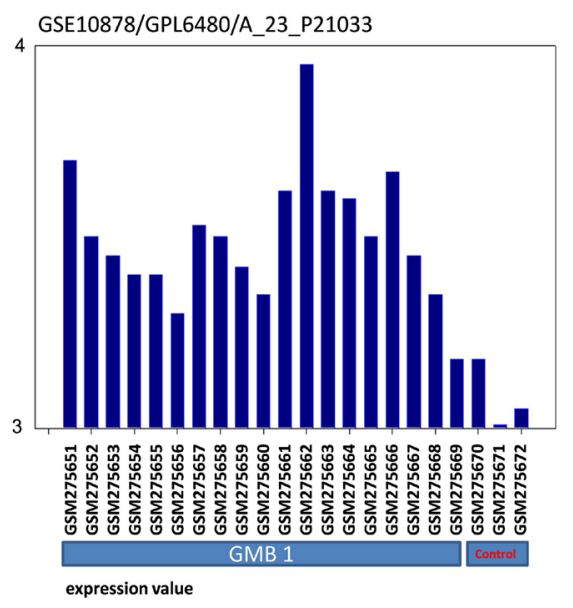

(a)
GSE10878/GPL6480/A_23_P155103

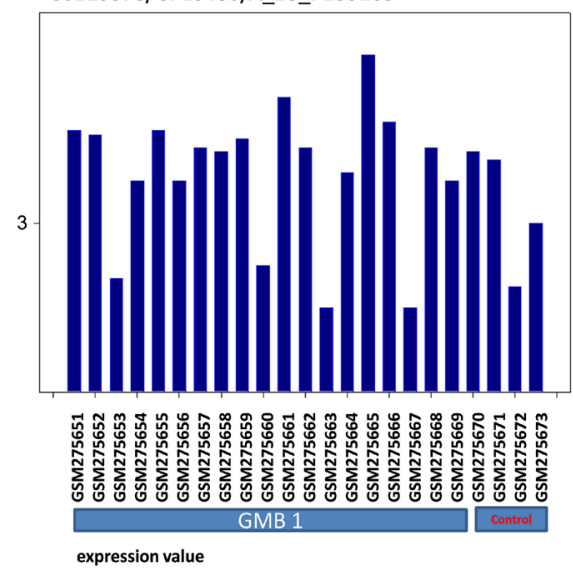

(b)

Figure 2. Intensity of the expression of the GMPS and ADSL in glioblastomavs normal tissue. A. GMPS profile. B. ADLS profile.

that they may arise from multiple cell types with neural stem cell-like properties. These cells are at multiple stages of differentiation from stem cell to neuron, to glia, with phenotypic variations determined, in large part, by molecular alterations in signaling pathways rather than by differences in the cell type of origin [19]. They might differentiate into the normal or atypical cells.

Mentioned above facts explain the existence of the common genes and gene sets, reflecting the geneses of the normal cells and development of the cancer and particularly the glioblastoma formations.

Delineation of the pathways related with the purine and pyrimidine metabolism in the settings of cancer once again is proving the fact of the intensive DNA syntheses in pathological settings.

As any biochemical pathway, purine and pyrimidine metabolisms's related reactions are determined and regulated by the activity of the rate-limiting enzymes.

These enzymes are hallmarks of the intensive DNA, RNA syntheses in the settings of the cancer and might serve as the diagnostic and predictive tools. From our data bases investigations it is clear, PRPS1 is the hallmark of the neurospheres formation, which might indicate on the early glioma development in the clinic. The GMPS and ADSL are representing the formed or generating gliomas.

\section{Conclusions}

We concluded and came to the following consequential results.

1) Neurogenesis and Glioblastoma are sharing some common genes.

2) Purine and pyrimidine metabolism-linked enzymes and genes are responsible for the upregulation of DNA and mRNA synthesis in the settings of the tumor development.

3) EGFR expression responsible genes, mRNA as well as proteins are up regulated during the development of the glioblastoma. 
4) GMPS genes are more strongly up regulated in the settings of the glioblastoma than ADSL.

5) PRPS1 is strongly synthetized in neurospheres than in the mature tissue during glioblastoma development.

\section{Conflicts of Interest}

The authors declare no conflicts of interest regarding the publication of this paper.

\section{References}

[1] Gyongyan, S.A., Manucharyan, T.G., Danielyan, K.E., Kevorkyan, G.A. and Chailyan, S.G. (2013) Xanthine Oxidoreductase Is a Key Enzyme of Purine Catabolism Regulation. Electronic Journal of Natural Science, 2, 17-21.

[2] Danielyan, K.E. and Kevorkian, G.A. (2011) Xanthine Oxidase Activity Regulates Human Embryonic Brain Cells Growth. Biopolymer and Cell, 27, 350-353. https://doi.org/10.7124/bc.000121

[3] Danielyan, K.E. (2013) Dependence of Cells Survival from Xanthine Oxidase and Dihydopyrimidine Dehydrogenase Correlative Activities in Human Brain Derived Cell Culture. Central Nervous System Agents in Medicinal Chemistry, 13, 108-113. https://doi.org/10.2174/1871524911313020003

[4] Aganyants, H.A., Abrahamyan, R.A., Chailyan, S.G., et al. (2014) New Highlights in the Regulation of Cells Proliferation. Neuroscience and Biomedical Engineering, 2, 81-86. https://doi.org/10.2174/2213385203666150213190534

[5] Wang, X., Yang, K. and Xie, Q. (2017) Purine Synthesis Promotes Maintenance of Brain Tumor Initiating Cells in Glioma. Nature Neuroscience, 20, 661-673. https://doi.org/10.1038/nn.4537

[6] Danielyan, K.E., Babayan, L.A. and Chailyan, S.G. (2019) Delineation of Effectors Impact on the Human Brain Derived Phosphoribosylpyrophosphate Synthetase Activity. Biomedical Journal of Scientific and Technical Research, 24, 17918-17926. https://doi.org/10.26717/BJSTR.2019.24.003988

[7] Danielyan, K.E., Vardanyan, R., Paronyan, Z.K., Barkhudaryants, I.M., Bisharyan, M.S. and Chailyan, S.G. (2018) PRPS-1 Is a Regulative for Neuroprotection and Cells Regenerative Proliferation. Journal of Biomolecules and Biochemistry, 2, 6-10.

[8] Lindsey, S. and Langhans, S.A. (2015) Epidermal Growth Factor Signaling in Transformed Cells. International Review of Cell and Molecular Biology, 314, 1-41. https://doi.org/10.1016/bs.ircmb.2014.10.001

[9] Gaestel, M. (2006) MAPKAP Kinases-MKs. Nature Reviews Molecular Cell Biology, 7, 120-130. https://doi.org/10.1038/nrm1834

[10] Vivanco, I. and Sawyers, C.L. (2002) The Phosphatidylinositol 3-Kinase AKT Pathway in Human Cancer. Nature Reviews Cancer, 2, 489-501. https://doi.org/10.1038/nrc839

[11] Niu, G., Wright, K.L., Huang, M., et al. (2002) Constitutive Stat3 Activity Up-Regulates VEGF Expression and Tumor Angiogenesis. Oncogene, 21, 2000-2008. https://doi.org/10.1038/sj.onc. 1205260

[12] Patterson, R.L., van Rossum, D.B., Nikolaidis, N., et al. (2005) Phospholipase C-Gamma: Diverse Roles in Receptor-Mediated Calcium Signaling. Trends in Biochemical Sciences, 30, 688-697. https://doi.org/10.1016/j.tibs.2005.10.005 
[13] Ye, D.Z. and Field, J. (2012) PAK Signaling in Cancer. Cellular Logistics, 2, 105-116. https://doi.org/10.4161/cl.21882

[14] Yu, P., Fan, Y., Qu, X., et al. (2016) Cbl-b Regulates the Sensitivity of Cetuximab through Ubiquitin-Proteasome System in Human Gastric Cancer Cells. Journal of BUON, Journal of Balkan Union of Oncology, 21, 867-873.

[15] Sigoillot, F.D., Berkowski, J.A., Sigoillot, S.M., et al. (2003) Cell Cycle-Dependent Regulation of Pyrimidine Biosynthesis. The Journal of Biological Chemistry, 278, 3403-3409. https://doi.org/10.1074/jbc.M211078200

[16] Danielyan, K.E. (2017) Egf Regulates Purine and Pyrimidine Metabolism. Journal of Biomolecules and Biochemistry, 1, In Press.

https://www.pulsus.com/journal-biomolecules-biochemistry/inpress.html

[17] Zollnerd, N. (1982) Purine and Pyrimidine Metabolism. Proceedings of the Nutrition Society, 41, 329-342. https://doi.org/10.1079/PNS19820048

[18] Thakkar, J.P., Dolecek, T.A., Horbinski, C., Ostrom, Q.T., Lightner, D.D., Barnholtz-Sloan, J.S. and Villano, J.L. (2014) Epidemiologic and Molecular Prognostic Review of Glioblastoma. Cancer Epidemiology, Biomarkers and Prevention, 23, 1985-1996. https://doi.org/10.1158/1055-9965.EPI-14-0275

[19] Phillips,, H.S., Kharbanda, S., Chen, R., Forrest, W.F., Soriano, R.H., Wu, T.D. and Aldape, K. (2006) Molecular Subclasses of High-Grade Glioma Predict Prognosis, Delineate a Pattern of Disease Progression, and Resemble Stages in Neurogenesis. Cancer Cell, 9, 157-173. https://doi.org/10.1016/j.ccr.2006.02.019 